\title{
Developing Decision Support Systems for Multiple Knowledge Domains: A Proposed Solution
}

\author{
Ninh T.U. Ho ${ }^{1}$, Luke Mirowski², Joel Scanlan ${ }^{1 *}$ \\ ${ }^{1}$ Discipline of ICT, University of Tasmania, Sandy Bay campus, Private Bag 51, Hobart, Tasmania, Australia. \\ ${ }^{2}$ Australian Forestry Operations Research Alliance (AFORA), University of the Sunshine Coast, Maroochydore, \\ Queensland.
}

* Corresponding author. Tel.: +61 36226 7825; email: joel.scanlan@utas.edu.au Manuscript submitted February 14th, 2019; accepted April 20, 2019. doi: $10.17706 /$ jcp.14.5.339-348

\begin{abstract}
With the emergence of the Internet of Things (IoT), there has been an exponential growth in the number of sensors and the data streams they produce. This sensor data is being used in a wide range of primary industries in Australia to enable smarter decisions at many levels of production. This data is only useful if it is accessible to decision makers in a timely manner. This paper examines how a framework that is applicable across multiple domains can be developed to enable rapid development of decision support tools. This aims to avoid development duplication and enable businesses to save time and money. The framework is aimed at rapidly producing Decision Support Systems (DSS) that can aggregate heterogeneous data streams into a single flexible architecture and visualize the data in a way that assists experts to make decisions.
\end{abstract}

Key words: Decision support, information systems, knowledge data engineering, sensor data.

\section{Introduction}

Many businesses in Australian primary industry have been developing their own specific Decision Support Systems (DSS) which enable decision makers to make better decisions in a timely manner in their industry contexts based on the data presented by real-time sensor systems. The result is DSSs which aligned to business practices at a decision-making level but at a technical level, there is duplication of effort from developers as they develop custom information systems based on the same underlying sensors technologies. As such, the objective of our study is to investigate the feasibility of using a multi-domain DSS for heterogeneous data streams from multiple disjointed sources within Australian primary industries. In short, leveraging the generic principles at the technology layers of DSS so that industry can rapidly produce DSS without duplicating developmental efforts, saving time and effort.

This paper starts by reporting on a review the previous work on DSSs in the context of architecture, characteristics and integrated components as well as a review of systems used globally to gain deep understanding of the knowledge requirements and the range specific DSSs (Section 2). Next, a methodology which defines and justifies an approach applied to conduct this study, including ethics issues is described in Section 3. Following, the next section reports the results of interviews with local industry stakeholders and then a set of Evaluation Criteria is established based upon successful DSSs in Environment, Marine, Agriculture, Aquaculture and Forestry industries combined with the expert experience and opinions. Section 5 introduces a solution for developing DSSs that specifically emphasises the software reuse and 
addresses reusability issues by building different functionalities of software as independent components or subcomponents that can be integrated into the software through a user interface. The final section discusses findings in relation to the need for an appropriate DSS and summarises the crucial outcomes of this study.

\section{Related Work}

Decision Support Systems (DSS) emerged in the 1970s in multiple fields aiming to utilise data from several different perspectives. One of the early concepts for DSS was defined as a management support system which relied on the interaction with computers that enabled managers to use data and models for analysing non-structured issues [1]. DSS has been described as an interactive computer-based system aiming to support users in addressing the poorly structured data and complex problems through a user interface where data and models, coupled with algorithms were combined [2]. DSS can facilitate users at any level of an organization to undertake strategic, tactical, and operational decision-making in a range of changing environments [3], [4].

A DSS is widely accepted as an interactive computer-based tool that enables users through computer communication utilizing data, knowledge, documents, and models to tackle problems and make decisions [5]. DSS tools often processes enormous amounts of data to provide a flexible, adaptable and interactive computer-based information system in order to tackle specific unstructured management problems [6].

The remainder of this section will review the core components of DSS and examine them in several knowledge domains to understand the need for a framework in multiple industry contexts.

\subsection{Architecture}

Architectures for developing DSS have been proposed and developed by many different researchers and developers [3], [5], [7]. These architectures describe DSS of consisting of three different fundamental components: databases, models, and a user interface. The latter acting as the communication module linking the other components and visualizing the target information to users who are also considered as a critical component of the architecture.

Although previous authors [3], [5], [7] showed similar ideas in relation to the typical components, there are the significant differences in the additional components and/or functionalities. Sprague [3] focused on the development of DSS which is used at all levels of management (strategic, tactical and operational planning), while Turban [7] built an Expert System (ES) that helps to create new models in a data component; and lastly, Power [5] designed that knowledge from experts is stored in the data component.

Additionally, other, more complex DSSs, require an additional component known as knowledge management that provides the knowledge for solutions of specific problems. It may integrate one or many ESs that act as a consultant and explain information to non-experts. The knowledge management system stores representations of descriptive, reasoning, procedural, and domain knowledge.

\subsection{Characteristics and Capabilities}

In the literature, there limited consensus in the definition or standard architecture of a DSS, as well as any standard characteristics and capabilities [6]. There are multiple theories or frameworks of DSSs and each one will be appropriate for particular domain of applicability, with examples in forestry, manufacturing or environmental management [8]. The key characteristics and capabilities of a relatively complete DSS synthesised in the study by Turban, Liang and Aronson [6] help DSS developers to have an overview of an appropriate DSS before developing any DSS. The key features of a DSS as well as brief descriptions, reported as in Table 1, are used to deeply review 41 existing DSSs. As a result, we can discover many of necessary characteristics and capabilities of a potential DSS that would be used in multiple 
domains.

\subsection{Evaluation Criteria}

A typical framework is developed based on components such as: data, model and user interface. Each framework had significant differences in the additional components and/or functionalities based on the particular need of each business and each industry.

Significantly, there are no consistent evaluation criteria of a successful DSS in any industry that have been published to date. As evaluation criteria are important to determine whether a framework is successful or not, in addition to proposing a reusable framework in multiple domains, this paper suggests evaluation criteria for our framework. To achieve this, we need to deeply understand DSSs used across multiple domains by in-depth focused reviewing and analysing of 41 DSSs which are already being used in some domains such as environment, marine, agriculture, aquaculture and forestry was conducted.

From the critical outputs of the deep review, the characteristics and capabilities of a successful DSS can be discovered and compared between different industries. The limitations of these systems will be analysed to propose a solution for developing DSS that can be used in multiple domains in a reusable, dynamic way. The outputs of this review are presented here in a summary form.

DSS in Environmental Management. 11 of publications of available technologies and models used in environmental and climate change management show that there have been varied predicted models and heterogeneous data used. Technological advances have allowed collecting of data about environmental conditions and their changes. Many of these DSSs collect a large amount of data; however, the detail of how this data is shared and used effectively has not been to be emphasised in these publications. In fact, visualising information should be an important feature of any DSS to help motivated decision makers use and develop such systems [9]-[11]. Furthermore, many sector specific models have been only used locally. Practically, these models should be plug and play in the whole field. Therefore, the point of a suitable framework is to accelerate the development of DSSs so that it is only the industry specific stuff that they need to add, such as the models, hat the entire rest of the DSS can be reused. As a result, the models will be used across the domains.

DSS in Marine Management. A review 9 of DSSs in this field showed us that a typical DSS in this field is integrated into a GIS in order to visualise data in its spatial context. A similarity between DSSs from environmental and marine management is the use of data and model components as two core parts of the systems. Additionally, the DSSs have been installed in mainframes and their use is limited because of the user interface and portability. Therefore, the user interface component is equally important in marine management. DSS developers also focus on the user features, such as interactive ease of use, effectiveness, and employed as a standalone or integration tool.

DSS in Agriculture Industry. A review 7 DSSs showed that most modern machinery systems have data collection features. Most growers have been increasingly using new technology that is able to automatically collect data and transfer it to a server creating an unprecedented huge volume of data; however, the next step as to how growers can access and use this source of data will become a critical issue in the future of the agricultural industry [12]. Most researchers are interested in how the data and the agronomic models they have studied are transferred to the farmers who use their results and findings in farming management decision-making. Therefore, besides the data and models, a potential DSS should have a friendly user interface that helps end-users to interact easily for supporting them in decision-making.

DSS in Aquaculture Industry. A review 7 DSSs showed that the majority of published papers emphasise building optimal models in fisheries and aquaculture sector rather than studying how aquacultists can apply effectively these models on their farms. There is little study of DSSs for fisheries and aquaculture as well as a lack of evaluation of these systems while the finding shows that many smaller DSSs have been well 
described about collection and management of heterogeneous data [13]. Additionally, most these systems focus on the collection of data and data analysis for modelling, and not the user interface. Thus, the study of how this data and the aquaculture models are transferred to the decision makers in aquaculture management could be an opportunity for further work in this knowledge domain.

DSS in Forestry Industry. A review 7 DSSs showed that some of the proposed and existing DSSs being used in this area have been considered to find similarities and differences among multiple domains. The DSSs of this industry have the similar architecture as that of other industries and especially the integration with GIS is very common. Besides data and modelling, the possibility of designing user interfaces has always been of interest.

Critical Outputs: The analysis of these systems reveals the available technologies and systems used in these sectors are extremely varied. However, there are still similarities among these systems about the collection of data and the use of models in decision-making. Many technological advances have allowed the collecting and sharing of data and most modern machinery systems also have data collection features. It can be clearly demonstrated that most DSSs focus on collection of data from a variety of sources using many advanced techniques. This leads to an unprecedented large volume of data being warehoused in these systems. However, the gap in existing studies that must be addressed is how decision makers can easily access and effectively use this data in a meaningful way. Another gap is the possibility of designing friendly user interfaces which allow end-users to interact easily with the data and the models or algorithms for supporting them in decision-making [12]. Transferring specific models to the end-user and the use of these across domains could be considered.

Table 1. The Key Characteristics and Capabilities of DSS

\begin{tabular}{|c|c|c|}
\hline \# & Features & Descriptions \\
\hline 1 & Semi/Un-structured problems & $\begin{array}{l}\text { Tackling semi-structured and/or unstructured situations through the combining } \\
\text { computerized information with human judgement }\end{array}$ \\
\hline 2 & All levels & Supporting all managerial levels from operation to strategy \\
\hline 3 & Individuals \& groups & Supporting both individuals and groups of people \\
\hline 4 & $\begin{array}{l}\text { Interdependent/ sequential } \\
\text { decisions }\end{array}$ & $\begin{array}{l}\text { Supporting interdependent and/or sequential decisions (once, several times, or } \\
\text { repeatedly) }\end{array}$ \\
\hline 5 & The entire process & Supporting in all steps of decision-making process \\
\hline 6 & Processes \& styles & Supporting a diversity of decision-making processes and styles \\
\hline 7 & Adaptable \& flexible & $\begin{array}{l}\text { Adding or modifying basic elements for dealing with quickly changing conditions and } \\
\text { solving similar problems }\end{array}$ \\
\hline 8 & Interactive ease of use & $\begin{array}{l}\text { Using a user interface with highly user-friendliness, strong graphical capabilities, and } \\
\text { a natural interactive }\end{array}$ \\
\hline 9 & Effectiveness & $\begin{array}{l}\text { Improving the effectiveness of decision-making, including accuracy, timeliness, } \\
\text { quality }\end{array}$ \\
\hline 10 & Human control & Supporting decision makers, not replacing them. \\
\hline 11 & Ease of development & Developing and modifying simple systems by end-users \\
\hline 12 & Modelling \& analysis & Models are generally utilized to analyze decision-making situations \\
\hline 13 & Data access & Accessing a diversity of data sources, formats, and types \\
\hline 14 & Standalone/ Integration & $\begin{array}{l}\text { As a standalone tool or distributed throughout an organization using Web and } \\
\text { networking technologies }\end{array}$ \\
\hline
\end{tabular}

\section{Methodology}

With the above analysis in mind, our study focuses on description and exploration the thoughts of domain experts in relation to their need and challenges of using DSSs. The study emphasises a close collaboration with participants to better understand the participants' views of reality in their organizational context and encourage the participants describing their requirements of potential DSSs. 
The purpose of our study was therefore to investigate the feasibility of a multi-domain DSS for heterogeneous data streams from multiple disjointed sources within Australian primary industries. We will describe how we have established the important characteristics associated with DSSs and how they can successfully assist decision-makers by firmly grounding it in the existing systems that have been reviewed above.

A qualitative method was used for identifying the requirements for, and potential usage of, a multi-domain DSS utilizing sensor data in Tasmanian primary industries. This qualitative case study approach was suitable in attaining as much information as possible from the participants' experiences and explored their in-depth understanding of their needs and challenges, while at the same time determining the suitability of a mobile application based DSS.

The study used semi-structured interviews and documentary evidence to understand the current issues and challenges of using DSSs. The interview transcripts were processed using the thematic analysis. The semi-structure interviews were approved by the Deputy Chair of the Tasmania Social Sciences Human Research Ethics Committee with Ethics Ref No: H0016070.

\section{Results}

The analysis of the interviews of domain experts from viticulture and forestry industry are reported in this section.

\subsection{Current Situation}

One of the key findings from the interviews was that most researchers in different fields share the almost same workflow. They use collected data to build many sector specific models. They are interested in how their findings or algorithms they have studied transferred to the end-user who is engaged in operating management decision-making. However, the transfer method differs depending on the conditions of the industry or individual of each researcher.

It is common for researchers upload their DSS-algorithms to a website while others built their DSS-models on Microsoft Excel or established the relationships among research data using SPSS.

A pipeline workflow of the academic works combined with their needs is proposed in Fig. 1 to help not only researchers understand the pipeline of their works and but also developers understand how a model or algorithm that assist growers in decision-making in their context can be built. Additionally, any DSS development -workflow is easy to develop as it can be seen clearly at the results of different roles and phases as the pipeline workflow presented.

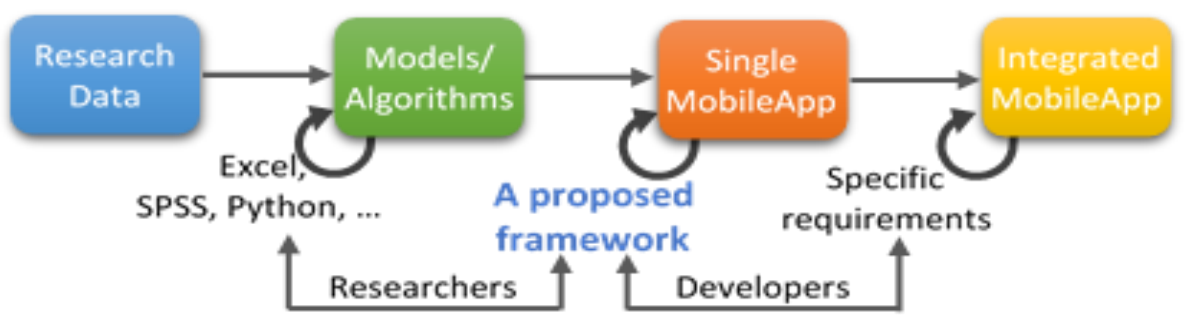

Fig. 1. A pipeline workflow of academic work from the interviews.

\subsection{Current Issues and Challenges}

End-users such as farmers, growers, and others often make decisions based on their experience rather than using decision-making tools. Significantly, the greatest challenge that experts in these industries have to face is how to connect and share data with the research sector. A prominent issue is how can researchers use industry data, and how can industry partners get access to new research results to improve 
productivity and efficiency in production.

Experts in viticulture reported that they are not sure about the quality of the collected data and argued that there are no criteria to judge whether the data is suitable, and its quality is good or not so far.

Technical challenges were also common, one expert reported that it is difficult to get data from various sources and integrate these types of data into one single dataset. For example, farmers access a weather server, log into software for this server and download data into their equipment. They then access into another website to get formulas of calculations the data and other information. This process is complicated. Hence, despite being provided with DSSs, growers will also stop using these tools when they can calculate manually by themselves or those tools are not useful.

Forestry experts reported that researchers have come up with tools, but that most of the tools are not actively used by the industry. A lot of tools that are used are older excel-based models; further there are paper-based tools that need to be quickly developed into applications that support decision-making in the industry, but currently they are disconnected from electronic DSS capabilities.

A common challenge is what type of device should be used for DSSs in the industries. End-users, such as timber harvesters, land owners, and others don't like to wait for the booting of a laptop, then launching the Excel application, inputting and waiting for the results to appear when they are on the fields, trucks or forests.

Next, one of the technical challenges in forestry industry is end-users usually use those simple tools to do their tracking and they are too busy to learn how to use complex tools or knowledge base in complex newer tools.

Finally, most researchers in this area find it is difficult to transform valuable data and models they researched into any application used by industry partners. Therefore, it is necessary to have a system that could integrate new models into an existing application in this area.

\subsection{Criteria for Success}

Some criteria for successful development of DSSs were recommended by the experts are synthesised in this section.

The first feature, namely Data Access, is highlighted in all papers as well as recommendations from the experts. The use of a variety of sources from The Bureau of Meteorology (BOM) or GIS to internal data with diverse formats and data types is an important element of a successful decision-making support system.

Similarly, the model component is equally important as it helps to transfer the gathered data to useful information and knowledge that is used by decision-makers. Most researchers want to put their research results, including models, formulas or algorithms, from academia to industry aiming to increase productivity and industry development. This is also a strong motivation for developing a multi-domain DSS for multiple disjointed sources data and a range of diverse models within Australian primary industries.

A DSS should provide accurate information in ease to view and in timely manner aiming to support and not to replace decision-makers. This feature reminds DSS developers to consider whether to build useful tools that are simple and easy to use, or complex systems with comprehensive support for users.

Normally, any DSS can be used as a standalone tool or distributed in an organization or among organizations along a supply chain through networking and web technologies. However, due to the specific nature of some industries, reachability and mobility are characteristics driving the interest of the experts. The decision-makers need access to information from any location at any time.

Therefore, DSSs developed on mobile devices are the top choice, which allow obtaining real-time data and providing useful information through visualization techniques from anywhere at any time. Besides that, some of experts said that it is necessary to integrate the potential tools and studies into the fields. Another important capability of a DSS is its flexibility and adaptability over time. This means that it allows users to 
add, remove, combine or change basic elements and can be easily modified to tackle other similar issues aiming to decision-makers dealing with rapidly changing environmental conditions. According to the expert, the maintenance and development of any DSS is important to make it always alive; thus, updating new models is necessary.

User-friendliness, powerful graphics capabilities with a naturally interactive user interface can increase the effectiveness of any DSS. Indeed, the experts argue that a potential tool should have a nice pretty user interface and easy to use [6].

Specifically, end-users always expect for any DSS to be nicer interface, easy-to-use and simple to do any task, while fully meeting their requirements for usability. Therefore, developers have to identify where and why the tools do not match and interview them more specific requirements.

Finally, the effectiveness of decision-making, including accuracy, timeliness and quality must be improved. Most experts claim that developing any DSS should be minimal cost while the tool must solve specific problems and correctly in prediction or calculation.

\subsection{Proposed Solution}

With the discussion of the interview results and the comparison between the current issues and the previous work, from above, a framework is proposed, and its components are described in this section. Broadly, the framework proposed here has a modular approach, adopted to design standardised components and subcomponents which are able to run standalone or integrate with each other. This allows developers to increase flexible levels of DSSs they build.

This proposed framework illustrated in Fig. 2 divides the managing data into two distinct parts, one is this data component that is considered as a database server and the other is an operational database of each DSS which is logically and physically separated from the server and other operational databases. The model component should store a collection of separate models that tackle with a distinct part of each issue.

This framework is flexible across knowledge domains, as it can be used to create a DSS tool, that is then customised by then incorporating the data models corresponding to issues of a particular industry, allowing for the tool to be modified for reuse easily in another industry. The operation component is any electronic device or software that manipulates data and performs many distinct functions through stand-alone or sharing networks.

The communication component may integrate many kinds of visualised techniques libraries, GIS or other systems into this system in order to make all components linked together and use easier, more flexible and quicker response. The user interface component provides an interface that enables users to interact with the system. Most flexibility and usability characteristics of a DSS are represented in this component.

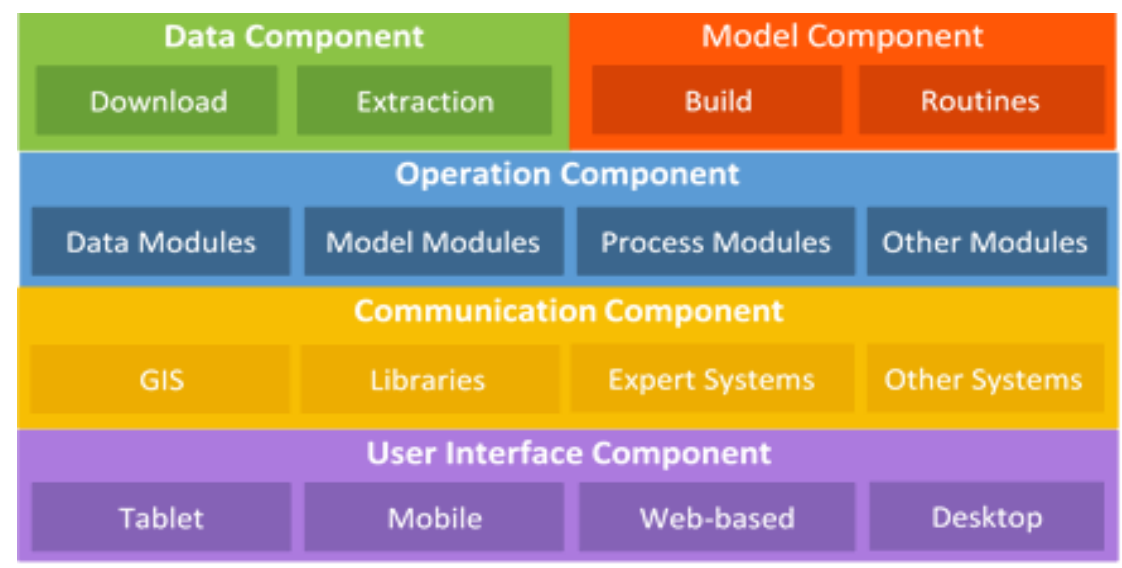

Fig. 2. A framework for developing DSSs in multiple knowledge domains. 


\section{Discussion and Conclusion}

From 1980 onwards there have been hundreds of frameworks for building a DSS for a particular industry. However, there has been no standard framework that can apply to multiple domains or any successful DSS has been used across multiple domains.

The particular frameworks in each domain seem to be not particularly helpful for many other industries because of their tight focus on the original domain of interest. It can be seen clearly that the development of DSSs has been still the traditional processes of developing software for a particular purpose as necessary.

Significantly, there are no evaluation criteria of a successful DSS in any industry that has been published to date. Although there are many DSSs used efficiently in many businesses of each industry, these systems are themselves commercial systems and often cost a lot to produce or purchase. It is difficult to establish evaluation criteria for the systems due to limited availability for research or observation by other industries.

In this study, much was learnt about specific industries such as environment, marine, agriculture, aquaculture and forestry. This study found that most typical DSSs generally have three different components, including data processing, model processing and a user interface with Excel-based or web-based being common. Some more modern systems are integrated with a geographic information system (GIS) and developed into mobile applications. One of the limitations of this study is that only 41 systems in five domains were assessed; therefore, the results of this assessment may not be replicated for many other existing systems. However, these results were used as the solid basis for interviewing industry experts and contributing significantly to the establishment of a framework that can be used to rapidly develop an application.

The findings found through expert interviews have brought the clearer points of view about the needs and challenges of using DSSs, as well as confirmed the right direction for this study. Once again, the need of developing and using DSSs has been confirmed as well as the difficulties that need to be addressed have been suggested by these experts. There are three major and urgent issues that need to be resolved.

Firstly, many experts and businesses have faced with the lack of data for use in their decision-making in the context of a sizeable amount of existing data that may be owned by themselves or their industry. An industry expert said that a huge amount of data collected does not currently get used in the Agriculture industry. The obvious fact is that it is difficult to get data from multiple sources and integrate it into a single source for examination. Secondly, the greatest challenge is to connect academia to industry. According to these experts, a researcher usually collects data from many sources, builds a model, wants to transfer this model to end-users who are likely use the model to support their decision-making, and get the feedback of these end-users about the status of the model. However, one of the reasons that these end-users made decisions based on their experience rather than using this model is the lack of usability of this model because of this paper-based or excel-based model. Additionally, end-users usually want to use simple tools to track their work and read the usefully needed information for their decision-making. Most end-users do not want to learn how to use complex systems or knowledge base in such tools. Therefore, many models produced by researchers are not used by the industry.

This study then introduced the use of a component-based software development approach to propose a framework for developing DSSs in multiple knowledge domains. The proposed framework is different from the previous work because of integrating all necessary components of the existing frameworks and adopting the modular approach. It provides a flexible structure in which its components or subcomponents are likely to independent of each other and are removable and assembled. This framework also allows integrating other systems into. It has the potential to assist software developers to easily analyse and systematically design any multi-domain DSS. This allows developers to increase flexible levels of DSSs they 
build.

Compared to the set of suggested evaluation criteria, this framework meets the criteria as follows. Firstly, the typical characteristics of a DSS with three components: data, model and user interface. Therefore, these components are main characteristics of this framework. Secondly, the evaluation criteria including human control, interactive ease for use and the effectiveness of decision-making are evaluated by the experts and these experts stated that these applications have the potential use and should be modified and extended for the future use. Significantly, one of the most important technical evaluation criteria of this framework is its flexibility and adaptability over time. This framework fully meets these seven original evaluation criteria. From the review of the literature in conjunction with the analysis of the experts' opinion, this study can be concluded that future trends in relation to multi-domain DSSs will continue to be a growing field of research. The framework presented in this study can be used in developing DSSs to enable easy reuse of any of its components and modules across multiple industries.

\section{References}

[1] Gorry, G. A., \& Scott Morton, M. S. (1971). A framework for management information systems. Sloan Management Review, 13, 55-70.

[2] Talluru, L. R., \& Deshmukh, A. V. (1995). Problem management in decision support systems: A knowledge-based approach. Proceedings of IEEE International Conference (pp. 1957-1962).

[3] Sprague, R. H. J. (1980). A framework for the development of decision support systems. MIS Quarterly, 4(4), 1-26.

[4] O'Brien, J. A., \& Marakas, G. M. (2011). Management Information Systems (10th ed.). New York, United States: McGraw-Hill/Irwin

[5] Power, D. J. (2002). Decision Support Systems: Concepts and Resources for Managers. London: Quorum Books Westport.

[6] Turban, E., Liang, T. P., \& Aronson, J. E. (2005). Decision Support Systems and Intelligent Systems (7th ed.). New York: Pearson/Prentice Hall.

[7] Turban, E. (1983). Decision support systems (DSS) a new frontier for industrial engineering. Computers and Industrial Engineering, 7(1), 41-48.

[8] Power, D. (2016). Retrieved from http://www.dssresources.com/interviews/ watson/watson11042005.html

[9] Al-Kassab, J., Quertani, Z. M., Schiuma, G., \& Neely, A. (2014). Information visualization to support management decisions. International Journal of Information Technology \& Decision Making, 13(2), 407-428.

[10] Schutt, R., \& O'Neil, C. (2014). Doing data science. O'Reilly Media, Sebastopol CA.

[11] Jongsawat, N., \& Premchaiswadi, W. (2011). A study of two different experimental settings for group awareness information in a web-based group decision support system. International Journal of Information Technology \& Decision Making, 10(2), 231-268.

[12] Somes, $\quad$ T. $\quad$ (2017). $\quad$ Retrieved from https://grdc.com.au/Media-Centre/Media-News/North/2017/01/How-digital-ag-and-big-data-can-he lp-growers

[13] Mathisen, B. M., Haro, P., Hanssen, B., Björk, S., \& Walderhaug, S. (2016). Decision support systems in fisheries and aquaculture: A systematic review. Aquaculture Engineering. 


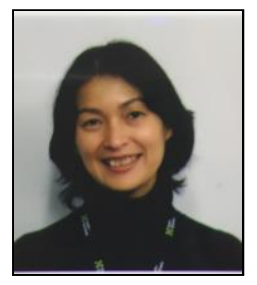

Ninh T.U. Ho has 19-year experience working as a software engineer for VNPT, the leading provider of telecommunication and IT services in Vietnam and for L\&K Technology Company in Vietnam. Particularly, her work focuses on development and implementation of software and systems using the on-demand programming languages.

She under took her masters at the University of Tasmania (UTAS) where her thesis focused on investigating and developing a flexible framework for rapid development decision support systems in multiple knowledge domains. She also worked as an academic tutor at UTAS.

Since April 2018, she has been working as an application developer of the Operational Technology Department, Hydro Tasmania. The department is charge of developing and maintaining technology to allow Hydro Tasmania to monitor and control energy generation assets and to operate in the national electricity market.

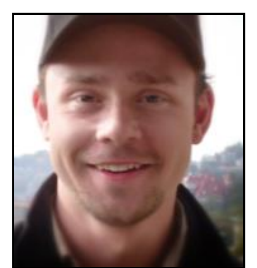

Luke Mirowski is a supply chain logistics consultant, senior research fellow in electronic logistics at the University of Tasmania (UTAS), and an adjunct research fellow to the Australian Forest Operations Research Alliance (AFORA) at the University of the Sunshine Coast (USC). He is a chartered member of the Chartered Institute for Logistics and Transport in Australia (CILTA). He has a formal background in computer science and logistics and has applied this expertise to investigate the ways in which software and sensor technologies can be integrated into supply chains for quality and productivity uplift.

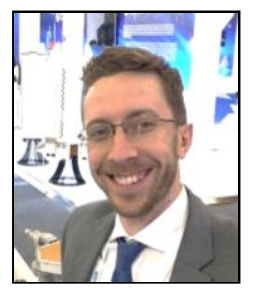

Joel Scanlan is from Tasmania Australia, where he completed all three of his computer science degrees, including his PhD. This was completed in the dual areas of cyber security and machine learning at the University of Tasmania.

Since completing his Ph.D, he has been an active researcher in a wide range of areas in an addition to security and machine learning. He has been very active in eHealth in relation to serious games for treating mental illness, as well as work relating to electronic health records. He has also undertaken work in relation to sensor systems in various industries, and their use within logistics supply chains. He is currently the cyber security lecturer at the University of Tasmania. 